\title{
Evaluation of severe trauma at the Emergency Department of the San Paolo Hospital of Savona: two years of experience
}

\author{
F. Frumento, L. Corti, A. Riccardi, G. Guiddo, P. Minuto, B. Chiarbonello, M. Castelli, R. Lerza
}

SC Pronto Soccorso, Osservazione Breve e Medicina d'Urgenza, Ospedale San Paolo, Savona

\begin{abstract}
The authors analyze the major trauma recorded in a couple of years in the Emergency Room of the San Paolo Hospital of Savona after the setting, in the same district, of the trauma center at the Santa Corona Hospital of Pietra Ligure. Despite the policy of centralization adopted the amount of major trauma seen at the San Paolo Hospital remained unchanged over time and the number of patients who are not rescued by medicalized ambulance is still significant. These observations underline the importance of periodically reviewing the protocols and make training on trauma also in hospitals that are not recognized as trauma center.
\end{abstract}

\section{Introduction}

In our district there are two major hospitals where the Emergency Departments are located: the Santa Corona hospital in Pietra Ligure (CTS, Centro traumatologico ad alta specializzazione - highly specialized trauma center) and the San Paolo Hospital in the city of Savona (PST, Pronto Soccorso per il trauma - first aid for trauma). The creation in 2009 of the trauma center at the hub center of Santa Corona highlighted the need for sharing of clinical care pathways on the centralization of major trauma. Physiological and anatomical criteria of identification of major trauma according to ATLS (1), were selected as basis for deciding when centralizing trauma patients, while other risk factors as dynamic of the accident were considered less important if present individually (Figure 1). So we decided to monitor the results of this approach by recording the patients with severe trauma observed at the spoke center of Savona in the two years following the creation of the trauma center. The study compare the two years of data collection recording the characteristics of patients, their outcome and changes induced by the centralized access protocol for severe trauma.

\section{Methods}

The study has provided for the registration of patients with major trauma observed in the San Paolo Hospital of Savona, in a period of two consecutive years from october 312009 to October 31, 2011. We considered only those patients who, at the end of the evaluation in the Emergency Room, had an injury severity score (2) of 15 or higher. The study excluded patients who were dead on arrival at the Emergency Room. In addition to the calculation of ISS, each patient was recorded according to sex, age, type of accident and its dynamic, mode of arrival at the hospital, outcome and hospitalization or transfer to other structure. Three months after the accident was carried out a telephone call to gather informations on the health status of patients especially with regard to outcomes. The data collected each year were compared with each other to assess the impact of the new organization and to reveal critical details.

\section{Results}

The number of patients with ISS greater than or equal to 15 was 35 in the first (group A) and 36 in the second (group B) year of observation. The main data recorded are shown in Table 1. In both groups of patients there was annual prevalence of male on female and road accidents on other types of accidents. The spectrum of age was very wide and this also reflects the fact that in our district there is a lot of very elderly people, mostly involved in domestic accidents. These last were approximately the $20 \%$ of the total. The percentage of immediate transfer of patients to the trauma center was similar in the two groups (31,4 versus $27,7 \%)$. Particularly relevant is the observation that only $57 \%$ of the patients in the group A and even $47 \%$ of the group B were rescued by ambulance with doctor on board (system 118). The remaining had reached the hospital transported by volunteers or by themselves. In group A 26 patients had the 
accident definitely threatening but only 18 of them were rescued and transported by ambulance with doctor on board. Also in group A we recorded 7 deaths within three months after the injury, thus direcly related to it. Five patients were permanently disabled. In group B a threatening dynamic of trauma involved 28 patients and only 15 of them $(53,7 \%)$ received rescue by a doctor out of hospital. In group B we recorded five deaths directly related to trauma and 8 permanent disability.

\section{Discussion}

The ISS is considered, in the international literature, one of the most sensitive ways to measure the major trauma and its consequences $(2,3)$. So we used this score to record the most serious trauma relief in our Hospital, and indeed we reached a significant number, but it is likely to assume that they could be more according to other methods. For example, using the New Injury Severity Score (4), it should be possible to detect, in comparison with ISS, a greater number of patients with score above 15. Other than that, the important element is that data here presented show that the observed annual number of severe trauma in the hospital of Savona remained constant despite the creation of the trauma center at about $20 \mathrm{Km}$ away. The explanation of this is probably in the kind of protocol of centralization adopted. Fearing an overestimation of the patients to centralize, the dynamic of the trauma was not considered as single risk factor for severe trauma, provided by our protocol. The result was that almost one third of the patients must be annually transferred from our Emergency Room to the trauma center, mainly for neurosurgical problems or spine surgery.

A recent paper (5) shows that a strict application of a protocol of centralization does not seem to cause an overload of patients to the trauma center, but only a better destination for them to the most appropriate treatments. Another aspect is particularly relevant to explain the lack of centralization of these patients, and this directly involves the rescue system. In fact many of these patients did not receive medical care in the territory having been rescued and transported to hospital by volunteers. They are ordered to transport the wounded to the nearest hospital and they can not take other decisions by themselves. Probably our rescue system can be improved in this respect. It's possible that a different filter of emergency calls allows a larger number of interventions of medically equipped ambulances. In these cases the physician, once the patient is evaluated according to established criteria, will decide where it is better to lead the wounded.

Our data confirm that the dynamic of the accident may just be an important criterion for prediction of severity of injury. In fact more than $70 \%$ of severe trauma here presented, had a relevant mechanism of injury recognizable by the history, and not regularly associated to anatomical or physiological findings. We can hypotize that a greater attention to this, will perhaps reduce the number of deaths and disabilities.

There are three other hospitals in addition to our that are defined "spokes" respect the hub of Santa Corona. They are located in areas with high population density that is in the town of Albenga, Imperia and Cairo Montenotte. So it is reasonable to predict a higher incidence of trauma in these areas than in the areas close to the trauma center. Then, if the criteria of centralization will not be extended, the spoke centers will receive paradoxically a number of serious injuries much higher than those in the hub center. Therefore mainly the peripheral centers will have to take care of first aid and stabilization of severely traumatized patients. Our data, collected for two years, seem to confirm this assertion and suggest the importance of trauma-specific training also for staff of more peripheral hospitals, because it is well established how important is the golden hour $(1,6)$ in the prognosis of major trauma.

\section{References}

1. American College of Surgeons Committee on Trauma. Advanced Trauma Life Support (ATLS) for Doctors. 2002 8th ed. American College of Surgeons.

2. Baker SP, O'Neill B, Haddon W Jr, Long WB. The injury severity score: a method for describing patients with multiple injuries and evaluating emergency care. J Trauma 1974; 14: 187-196.

3. Stevenson M, Segui-Gomez M, Leschoier I, Di Scala C, McDonald-Smith G. An overview of the injury severity score and the new injury severity score. Injury Prevention 2001; 7: 10-13.

4. Osler T, Baker SP, Long W. A modification of the injury severity score that improves accuracy and simplifies scoring. J Trauma 1997; 43: 922-925.

5. Melcarne L, Ricciardelli A, Melandri R, Farinatti M, Gatti A, Occhionorelli S. La centralizzazione del politrauma. Studio della realtà ferrarese e simulazione della presenza di un protocollo concordato. Emergency Care Journal 2011; 3: 15-19.

6. Lerner EB, Moscati R. The golden hour: scientific fact or medical "urban legend"? Acad Emerg Med 2001; 8: 758760. 